\title{
A 16PSK-OFDM-FSO Communication System under Complex Weather Conditions
}

\author{
Junyi Zhou, Yufeng Shao, Zhifeng Wang, Changxiang Li, Yue Zhou, Wenzhe Ma \\ Faculty of Engineering, Shanghai Polytechnic University, Shanghai, China \\ Email: yfshao@sspu.edu.cn
}

Received 2 July 2016; accepted 18 August 2016; published 25 August 2016

\begin{abstract}
Free Space Optical (FSO) communication is one of the most promising access methods. But, the different weather conditions will affect the transceiver performance. In this paper, we build a FSO communication system with 16-ary phase shift key-orthogonal frequency division multiplexing (16PSK-OFDM) downstream signals. We analyze the transmission performance of 16PSK-OFDM optical signals under five different weather conditions (sunny, light rain, moderate rain, snow, and fog). The optical spectral diagrams, constellation diagrams, and bit error rate (BER) of the received signals are simulation measured and analyzed. The results show that the constellation diagram under sunny condition is clear, and the value of BER is about $10^{-4.2}$ after 16PSK-OFDM optical signals pass through $500 \mathrm{~m}$ FSO link. Note that, the constellation points are dispersed under the other weather conditions.
\end{abstract}

\section{Keywords}

Optical Communication, OFDM, FSO, BER, Constellation Diagram

\section{Introduction}

Free space optical (FSO) communication is a technique of using laser beam as an information carrier, to realize point-to-point or point to multi-points transmission. Its advantages such as large capacity, good security, strong anti-interference ability, no frequency applications permission, make FSO become a flexible system for broadband access. Therefore, FSO has been widely concerned in the fixed wireless broadband access technology [1]-[3]. However, the FSO channel is a complex channel, which is influenced by atmospheric absorption, scattering and turbulence. In broadband wireless access systems, orthogonal frequency division multiplexing (OFDM) modulation technology can overcome some negative effects, and it will be the best choice for the next generation of wireless access system [4]-[6]. Moreover, the generated optical signals can be transmitted successfully in the atmosphere channel [7]. The high order modulation can make full use of the bandwidth of the channel and improve the anti-noise ability in FSO systems [8] [9]. Therefore, we design a FSO communication system based on 16-ary phase shift keying orthogonal frequency division multiplexing (16PSK-OFDM).The simulation experiment is carried out, and the influence of different weather conditions to FSO systems is analyzed, and the obtained results prove FSO is a potential broadband access choice in future [10]. 


\section{16PSK-OFDM-FSO Communication System}

As is shown in Figure 1, the 16PSK-OFDM-FSO communication system can be divided into transmitter and receiver. Transmitter is mainly composed of OFDM modulation module, electric/optical conversion module and FSO channel. Receiver is mainly composed of OFDM demodulation module and optical/electric conversion module. The main function of OFDM modulation module is to transforms data bits into OFDM symbols, which produce the base band time series signals. The main function of the electric/optical conversion module is transforms the OFDM linearly energy spectrum from the radio frequency domain to the optical domain, which is suitable for the transmission in free space. OFDM signals through the FSO channel arrive at receiver end. After signals passed through the Gauss filter, the demodulation process is carried out at the opposite of the transmitter end, thereby the original transmission signals are restored.

The electric/optical conversion module is shown in Figure 2(a), which converts electrical signals to optical signals by using two optical I/Q modulators. Optical I/Q modulator is composed of two Mach-Zehnder Modulator (MZM), one MZM is used for generating the I component, another MZM and 90 degree phase shifter areused for generating the $\mathrm{Q}$ component. The electrical signals are divided into I port and the Q port of the optical I/Q modulator and converted to optical signals.

The optical/electrical conversion module is shown Figure 2(b), the OFDM coherent optical signals are converted to electrical signals by using one balanced dual detector heterodyne detection. The dual balance detector is made up by two pairs of PIN photoelectric detectors. In order to detect in-phase and quadrature components of

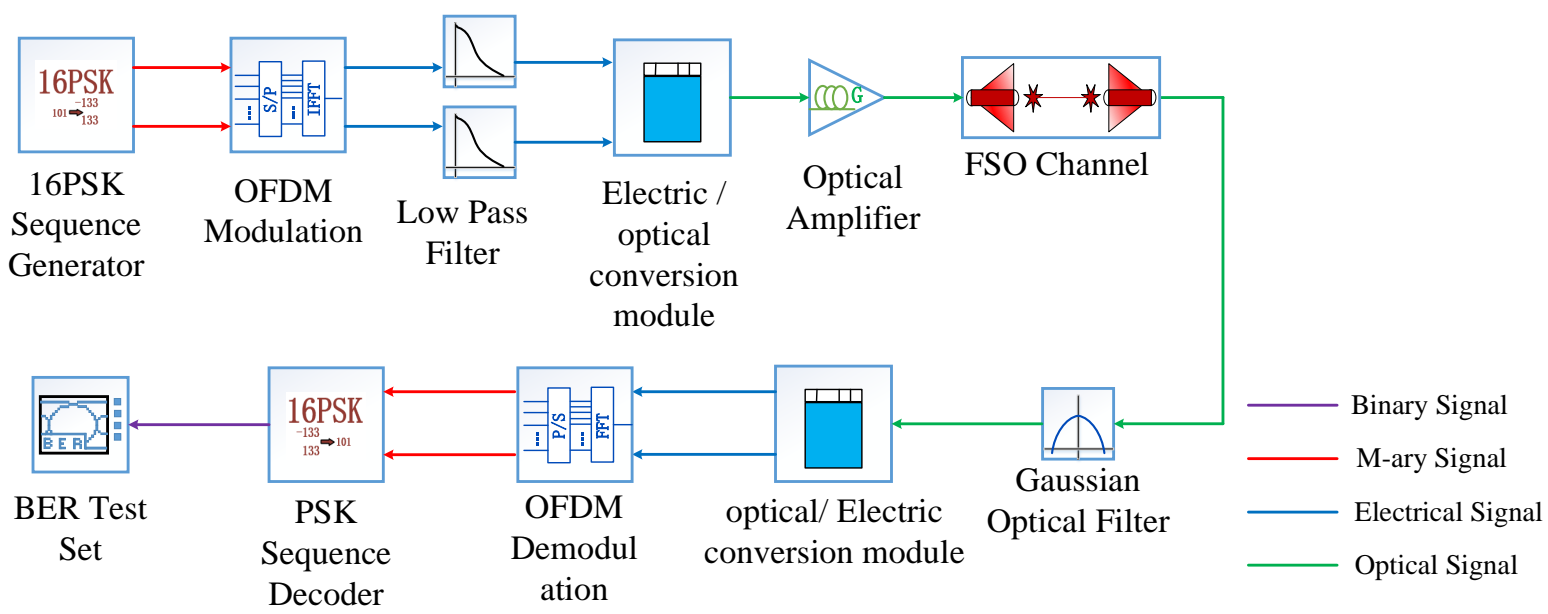

Figure 1. Block diagram of 16PSK-OFDM-FSO system.

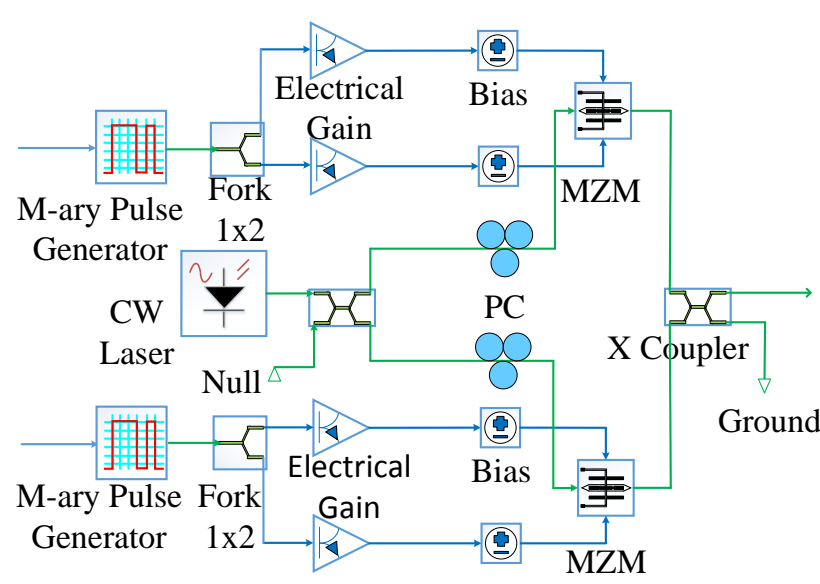

(a)
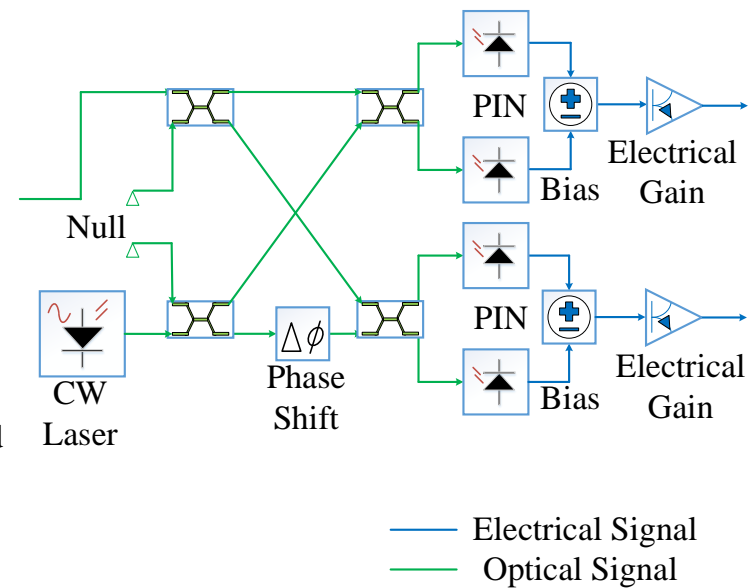

(b)

Figure 2. System configuration. 
the OFDM signals, the phase difference between the input vibration signals of the dual detector is 90 degrees. This module convert the mixing light field of local oscillation signals and optical signals into electrical signals. The amplification of the optical signals are realized by a strong local oscillator, the signal to noise ratio of the system is improved, the sensitivity of receiver is improved, and the link distance is increased effectively.

The specific parameters of the devices shown in Figure $\mathbf{2}$ are as follows: The frequency of continuous wave laser is $193.1 \mathrm{THz}$, the line width is $0.1 \mathrm{MHz}$, the coupling power is $5 \mathrm{dBm}$, the extinction ratio of MZM is 60 $\mathrm{dB}$, the sensitivity of PIN photoelectric detector is $1 \mathrm{~A} / \mathrm{W}$, and the dark current is $10 \mathrm{nA}$.

FSO-OFDM is a point to point laser communication system, when the laser passes through the atmospheric channel, it will be subject to the interference of the dust, smoke, fog, water and other solid, liquid and gas suspended particles in the channel. The scattering effect of the atmosphere on the laser signals is related to the number and size of the particles, the number of particles increases, and the scattering attenuation is more serious. With the weather changes of cloud, rain, snow and fog, the scattering attenuation caused by the particles in the atmosphere is also different. In different weather conditions, the amplitude and phase noise of the signals are brought to a different extent. This will affect the communication quality, shorten the communication distance, and even lead to the communication interrupt. In the sunny, cloudy, rain, snow, and fog weather conditions, attenuation values are shown in Table 1.

The FSO channel module is composed of a transmitting telescope, free space and a receiving telescope. The attenuation of laser power is mainly determined by three main parameters: atmospheric attenuation, geometric loss, transmission distance between transmitting and receiving telescope. The relationship between the received power and the transmit power can be expressed as:

$$
P_{\text {received }}=P_{\text {transmitted }} \frac{d_{R}^{2}}{\left(d_{T}+\theta R\right)^{2}} 10^{-\alpha \frac{R}{10}}
$$

Formula: $d_{R}$ represents the receiving aperture diameter $(\mathrm{m}), d_{t}$ representative of the transmit aperture diameter (m), $\theta$ represents the beam divergence angle (ARC), $\mathrm{R}$ represents the distance $(\mathrm{km}), \alpha$ represents of the atmospheric attenuation $(\mathrm{dB} / \mathrm{km})$.

The user can also set the corresponding parameters according to the transmission loss and the receiving loss of the transmitting telescope and the receiving telescope. If the intensity flicker parameter is enabled, the Gama-Gama distribution is used to simulate the atmospheric attenuation. In this case, the probability $I$ of a given strength is:

$$
P(I)=\frac{2(\alpha \beta)^{(\alpha+\beta)}}{\Gamma(\alpha) \Gamma(\beta)} I^{(\alpha+\beta) / 2-1} \mathrm{~K}_{\alpha-\beta}(2 \sqrt{\alpha \beta I})
$$

Formula: And represent the variance of large and small turbulence respectively, $\Gamma()$ is the Gama-Gama distribution function, and $\mathrm{K}_{\alpha-\beta}()$ is the second form of the improved Basel function.

$$
\alpha=\exp \left[\frac{0.49 \sigma_{R}^{2}}{\left(1+1.11 \sigma_{R}^{1 / 5}\right)^{5 / 6}}\right]-1 \quad \beta=\exp \left[\frac{0.51 \sigma_{R}^{2}}{\left(1+0.69 \sigma_{R}^{12 / 5}\right)^{5 / 6}}\right]-1
$$

\section{Simulation Results Analysis}

A long-term outdoor experiment based on 16PSK-OFDM-FSO system is carried out, five kinds of typical weather data are selected from the experimental data in a large number of different weather conditions, and the optical spectral diagrams, constellation diagrams, and BER of the received signals are compared and analyzed.

Table 1. Transmission losses in 5 typical weather conditions.

\begin{tabular}{cc}
\hline Weather & Attenuation \\
\hline Sunny & $3 \mathrm{db} / \mathrm{km}-7 \mathrm{db} / \mathrm{km}$ \\
Light rain & $7 \mathrm{db} / \mathrm{km}-15 \mathrm{db} / \mathrm{km}$ \\
Moderate rain & $25 \mathrm{db} / \mathrm{km}-40 \mathrm{db} / \mathrm{km}$ \\
Snow & $100 \mathrm{db} / \mathrm{km}-150 \mathrm{db} / \mathrm{km}$ \\
Fog & $200 \mathrm{db} / \mathrm{km}-300 \mathrm{db} / \mathrm{km}$ \\
\hline
\end{tabular}


Atmospheric channel is a kind of time varying channel with memory, and atmospheric scattering has a great influence on the constellation diagram of the modulated signals, and it is difficult to detect the phase ambiguity, offset and Doppler shift. The influence of different weather on the optical signals transmission are not the same, the spectrum of the signals in the receiver is shown as the power down, the image is lost and deformed. In the constellation diagram, the vector point dispersion, rotation, deformation and so on. The above Figure 3 and Figure 4 are 100 meters, 500 meters after the atmospheric channel transmission of 16PSK optical signals under different weather conditions. It can be seen that the signals are different from the degree of attenuation under different weather conditions. Even in the same weather conditions, the transmission distance will greatly affect the reception power of the signals.

As is shown in Figure 5, the effect of sunny days on the transmission quality is minimal. Cloudy, rain, snow, fog, impact on the transmission quality increased. When the weather is sunny, the FSO channel is more stable,
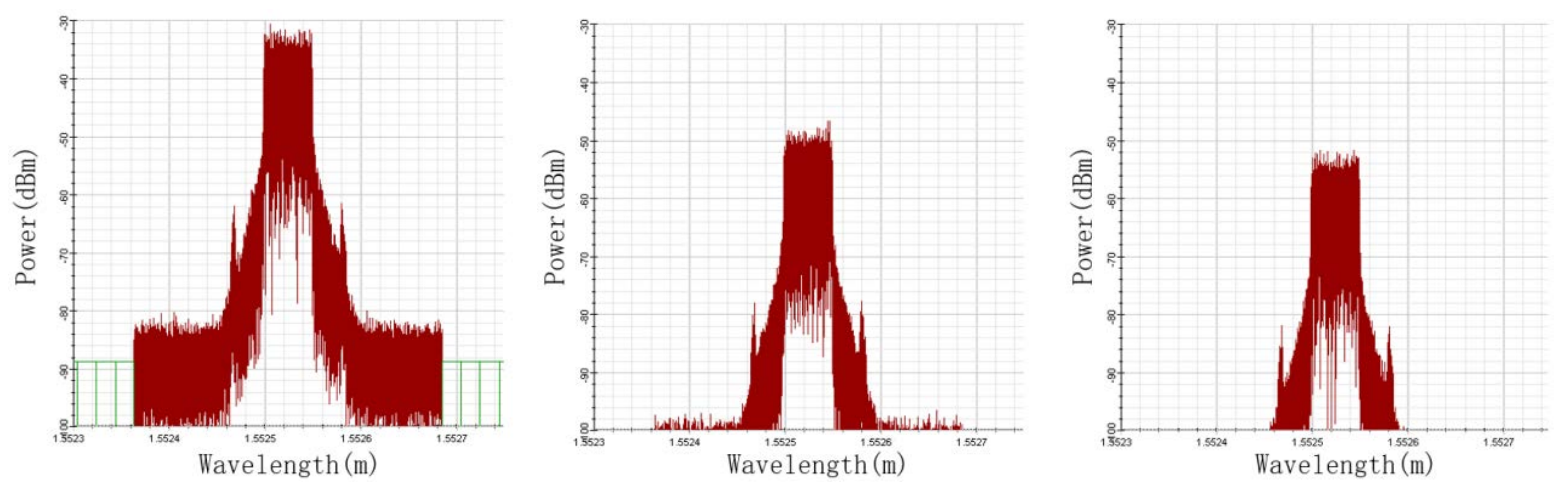

Figure 3. Spectrum diagrams of the received signals transmitted 100 meters under different weather.
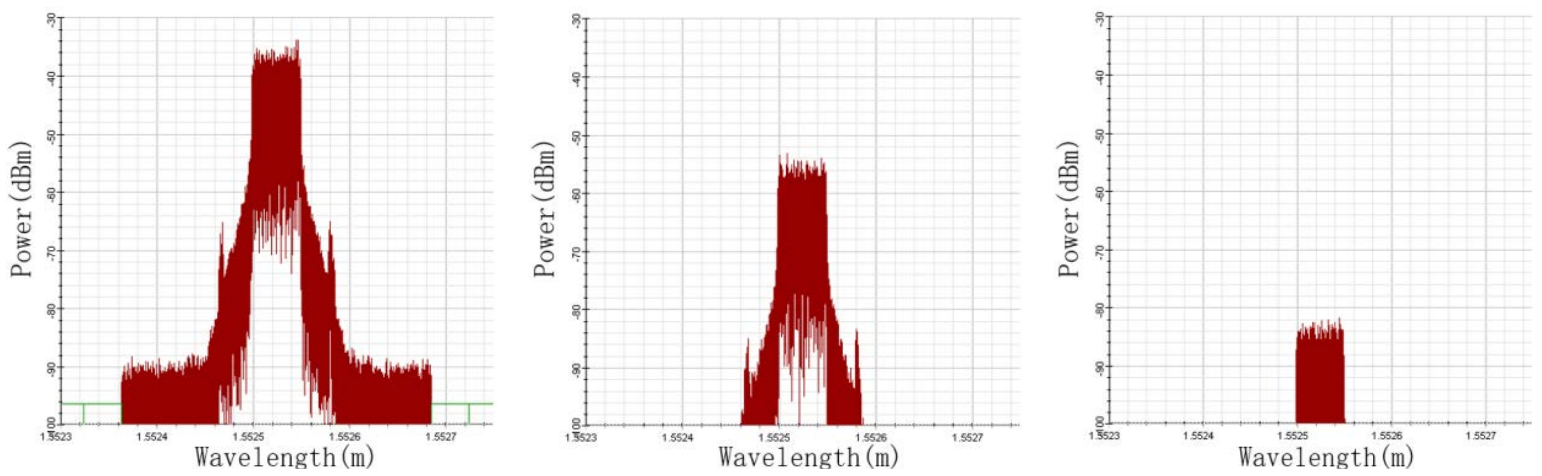

Figure 4. Spectrum diagrams of the received signals transmitted 500 meters under different weather.
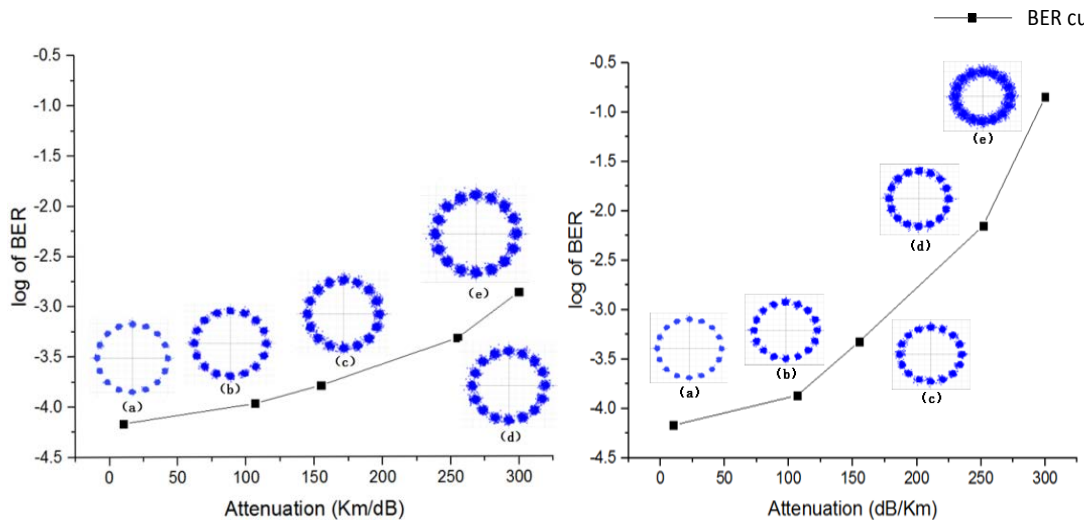

Figure 5. The BER curves of 16PSK-OFDM optical signals (FSO links are $100 \mathrm{~m}$ and $500 \mathrm{~m}$ ). 
the constellation points in the 2 dimensional space is basically evenly distributed into 16 concentrated regions, the constellation points without aliasing, and the distribution is more concentrated. Light signals are mainly affected by the scattering of suspended particles in the atmosphere, absorption of molecules, and the effect of atmospheric turbulence, but less affected by the atmospheric attenuation. The scattering effect of the atmosphere is related to the number and size of the particles, the more particles, the more serious of scattering, the greater of the attenuation it is. The density of fog and rain is larger in rainy days, part energy of the light beam is scattered in all directions. This forms the scattering effect. So the effect of light rain on amplitude and phase information of optical signals is less than moderate rain. As is shown in Figure 5(b) and Figure 5(c).Constellation diagrams of the received signals are shown in Figure 5(e), distribution of vector points in the constellation diagram is scattered, the constellation diagram is presented as a fuzzy ring, and we cannot distinguish the phase information. So the effect of fog on optical signals is extremely serious. Signals BER is the largest in the fog, reaching $10^{-2.15}$, and the lowest in the sunny, is $10^{-4.16}$. The BER of light rain and moderate rain were $10^{-3.32}$ and $10^{-3.69}$, respectively.

\section{Conclusion}

An effective implementation scheme of 10-Gb/s16PSK-OFDM-FSOsystem is proposed and analyzed. A short range communication link simulation is carried out under different weather conditions. The optical spectral diagrams, the constellation diagrams, and the BER performance of the received signals are measured at the receiver. With weather conditions change from cloudy, rain to fog, the receiver sensitivity is degraded. In the fog day, the divergence degree of constellation is seriously and the phase information is fuzzy. The simulation results show, the 16PSK-OFDM-FSO system has good transceiver performance and feasibility for user's access. Hence, FSO system application is a good solution scheme for long distance, large capacity and flexible access.

\section{Acknowledgements}

This work is partially supported by the National Natural Science Foundation of China (No. 61107064), Innovation Program of Shanghai Municipal Education Commission (No. 15ZZ101), Leading Academic Discipline Project of Information and Communication Engineering (No. XXKZD1605), School Foundation (No.

EGD14XQD01) of Shanghai Polytechnic University, College Students' Science and Technology Innovation Project of Shanghai Polytechnic University (No. 2016-xjkj-063), and College Student Innovation Activity Plan in Shanghai (No. 2013-sj-cxjh-028).

\section{References}

[1] Chen, D., Ke, X.Z., Zhang, T. and Lu, D. (2015) Experimental Research on Wireless Optical Communication Based on 16PSK Sub-Carrier Modulation. Chinese J Lasers, 42, 197-204.

[2] Zhao, L. and Ke, X.Z. (2009) A FSO-OFDM Model Based on Time Frequency Code. Chinese J Lasers, 36, 27572762.

[3] Cvijetic, N., Qian, D.Y. and Wang, T. (2008)10 Gb/s Free Space Optical Transmission Using OFDM. Conference on Optical Fiber Communication/National Fiber Optic Engineers Conference.

[4] Ohtsuki, T. (2008) Multiple-Subcarrier Modulation in Optical Wireless Communication. IEEE Communication Magazine, 41, 74-79. http://dx.doi.org/10.1109/MCOM.2003.1186548

[5] Guo, Q., Tran, A.V. and Chae, C.-J. (2011) 10-Gb/s WDM-PON Based on Low Bandwidth RSOA Using Partial Response Equalization. IEEE Photonics Technology Letters, 23, 1442-1444. http://dx.doi.org/10.1109/LPT.2011.2162824

[6] Shao, Y.F., Wang, Y.J. and Chi, N. (2013) 60-GHz RoF System with Low PAPR 16QAM-OFDM Downlink Using PTS Segmentation. IEEE Photonics Technology Letters, 25, 855-858. http://dx.doi.org/10.1109/LPT.2013.2252425

[7] Shao, Y.F., Zhang, J.W., Fang, W.L., Huang, B., Zhang, Z.R. and Tao, L. (2010) DRZ, DQPSK, and PoISK Orthogonal Modulations for 100Gbit/s Transmission System Applications. Asia Communications and Photonics Conference and Exhibition, 142-143. http://dx.doi.org/10.1109/ACP.2010.5682795

[8] Seo, J.-H., Seo, Y.-K. and Choi, W.-Y. (2006) 1.244-gb/s Data Distribution in 60-GHz Remote Optical Frequency UpConversion Systems. IEEE Photonics Technology Letters, 18, 1389-1391.

[9] Heine, F., Kämpfner, H., Czichy, R., Meyer, R. and Lutzer, M. (2010) Optical Inter-Satellite Communication Operational. Military Communications Conference, 1583-1587. http://dx.doi.org/10.1109/milcom.2010.5680175

[10] Yi, X.W., Shieh, W. and Ma, Y.R. (2008) Phase Noise Effects on High Spectral Efficiency Coherent Optical OFDM Transmission. Journal of Lightwave Technology, 26, 1309-1316. http://dx.doi.org/10.1109/JLT.2008.919368 


\section{Submit or recommend next manuscript to SCIRP and we will provide best service for you:}

Accepting pre-submission inquiries through Email, Facebook, LinkedIn, Twitter, etc.

A wide selection of journals (inclusive of 9 subjects, more than 200 journals)

Providing 24-hour high-quality service

User-friendly online submission system

Fair and swift peer-review system

Efficient typesetting and proofreading procedure

Display of the result of downloads and visits, as well as the number of cited articles

Maximum dissemination of your research work

Submit your manuscript at: http://papersubmission.scirp.org/ 